I NRA Prod. Anim., 2001, 14 (2), 97-103
D. EDDERAI, M. NTSAME, P. HOUBEN

Vétéri nai res Sans Fronti ères - Projet DGEG, BP 9129, Li breville, Gabon

Courriel : vsfgab2@internetgabon.com

\section{Gestion de la} reproduction en aulacodiculture. Synthèse des outils et méthodes existants

L'aulacode est un rongeur sauvage qui vit exclusivement en Afrique. Sa viande est très appréciée par les consommateurs de gibier. Le développement de son élevage, depuis une quinzaine d'années, répond à des préoccupations concernant la protection de la faune, le contrôle du commerce de la viande de brousse et la recherche d'alternatives locales à l'importation de viande. Comme pour toutes les espèces, la maîtrise de la reproduction est un facteur important de la productivité des élevages.

L'aulacode (Thryonomys swinderianus, Temminck 1827 ; photo 1) est un rongeur exclusivement africain, appartenant au groupe des Hystricomorphes. Les mâles adultes pèsent de 4,5 à $7 \mathrm{~kg}$ et les femelles de 2,5 à 4,5 $\mathrm{kg}$. On le rencontre dans toute I'Afrique subsaharienne au sud du 15ème parallèle Nord jusqu'au Cap en passant par le centre de la Namibie (figure 1 ; Kingdon 1997).

Sa chair est très prisée par les consommateurs africains de viande de brousse, qu'ils soient citadins ou ruraux. C'est pourquoi, depuis plus de 15 ans, divers projets en Afrique de l'Ouest et en Afrique Centrale se sont penchés sur la mise au point et la diffusion de techniques d'élevage à son égard (J ori et al 1995). C'est dans ce cadre que s'inscrit le projet "Développement au Gabon de l'E levage de Gibier" (DGEG), mis en œuvre par I'association Vétérinaires Sans Frontières et financé par I'Union Européenne.

\section{Résumé}

L'élevage d'aulacodes est aujourd'hui techniquement bien maîtrisé, et la reproduction en captivité de ce rongeur africain ne pose plus de problème. Toutefois, dans une logique économique, une bonne gestion de la reproduction est indispensable afin de permettre une productivité optimale des femelles. Dans ce cadre, il est important que les géniteurs soient identifiés, afin d'éviter les croisements consanguins et d'assurer une sélection des individus les plus performants. La reproduction a lieu en groupes polygames, les femelles, plus légères que le mâle, étant transférées dans l'enclos de celui-ci. Un test de gestation simple et précoce par examen du mucus vaginal est disponible et sa pratique doit être généralisée. Les femelles gestantes peuvent alors être isolées une à une jusqu'au sevrage des jeunes (mise en accouplement temporaire), ou au contraire laissées en permanence avec le mâle dans l'enclos (mise en accouplement permanent), qui permet une saillie post-partum très précoce.
Aujourd'hui, les techniques de base de l'aulacodiculture sont parfaitement connues (Van de Velde 1991, Productions animales 1992, Jori et Noël 1995, Schrage et Togbe Yewadan 1995, Heymans 1996) et le nombre d'aulacodiculteurs va sans cesse croissant avec maintenant plus de 700 élevages recensés en Afrique de I'Ouest et Centrale. Toutefois, à mesure que l'élevage d'aulacodes tend à se développer sur un modèle intensif, la maîtrise de certains facteurs de production, dont la gestion de la reproduction, se pose comme un impératif afin d'optimiser la productivité et la rentabilité de cet élevage.

Sur la base de l'expérimentation développée par le DGEG depuis 1995 au sein de sa station de multiplication située à Owendo en périphérie de Libreville, d'une part, et de l'expérience des éleveurs gabonais d'autre part, cet article se propose de rappeler les caractéristiques de la reproduction chez l'aulacode, d'exposer les pratiques nécessaires à une bonne gestion de la reproduction, puis de présenter les deux modes de mise en accouplement couramment appliqués en aulacodiculture et de proposer des améliorations que I'on pourrait apporter à chacun d'eux.

\section{1 / Caractéristiques de la reproduction}

Chez les aulacodes, la puberté est atteinte dès l'âge de 4 mois. Les femelles pèsent alors en moyenne $1515 \mathrm{~g}(n=353)$ et les mâles $1711 \mathrm{~g}$ $(n=263)$. L'âge pour la mise à la reproduction doit cependant être un peu plus élevé : 6,5 mois pour les femelles et 8 mois pour les mâles. 
Photo 1. Aulacode adulte.

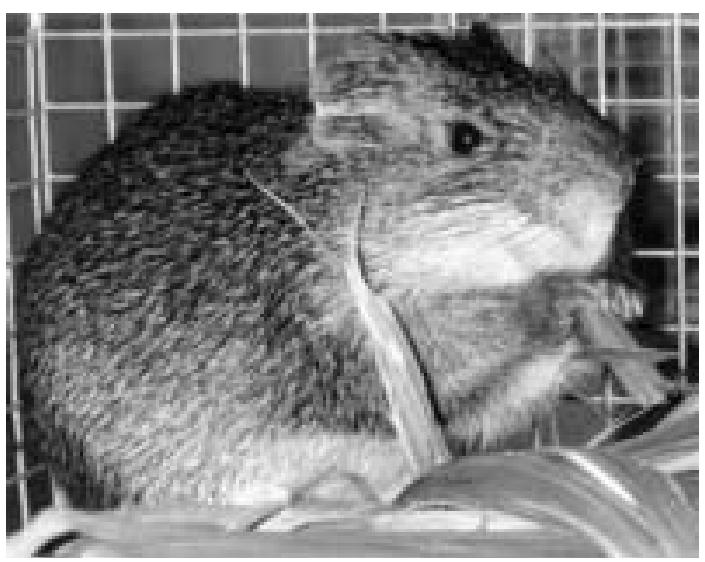

Dans des conditions d'élevage où le disponible fourrager est constant toute l'année et où la photopériode varie peu, la reproduction a lieu toute l'année.

L'œstrus est déclenché par la présence du mâle et par la cour assidue qu'il fait à la femelle (Adjanohoun 1992). L'ovulation est multiple et répond à un mécanisme réflexe déclenché par le coït, qui ne peut avoir lieu "mécaniquement" que durant l'oestrus. (Adjanohoun 1992). Toutefois, les ovocytes fécondés ne donneront pas tous de futurs fœtus. II existe en effet une période critique chez la femelle gestante entraînant une résorption embryonnaire partielle avec saignements, à partir du 30ème-35ème jour de gestation. Ce phénomène traduit une baisse temporaire du taux plasmatique de progestérone dû à l'involution des corps jaunes ovariens (Adjanohoun 1988).

En l'absence de gestation, la vulve de la femelle est généralement fermée par une croûte jaune ou marron clair. Le mucus vaginal est jaunâtre, blanchâtre ou transparent. $\mathrm{Au}$ moment de la gestation, la croûte vaginale cède la place à une membrane vaginale qui se confond avec l'épithélium cutané. Le mucus quant à lui se transforme en un mucus particulier appelé " bouchon vaginal de gestation ", très collant et très élastique (Adjanohoun 1988).

Chez la femelle aulacode, la gestation dure $152 \pm 2$ jours (Mensah et Baptist 1986). Cette
Figure 1. Distribution géographique de l'aulacode (Thryonomys swinderianus).

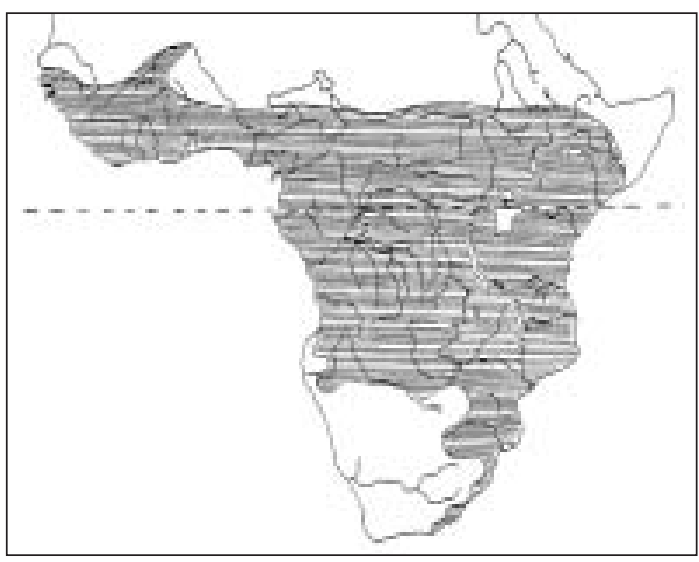

longue durée de gestation est un phénomène commun chez les Hystricomorphes (Weir 1974).

Le nombre de jeunes par portée varie de 1 à 13 et est en moyenne de 5 (DGEG 1999). La prolificité des femelles peut être amoindrie en cas de mise à la reproduction trop précoce, mais n'est pas affectée par l'âge. Toutefois, il semble que l'espérance de vie des aulacodes d'élevage se situe entre 4,5 et 6 ans. II est donc recommandé de réformer les reproducteurs vers 4,5 ans.

\section{2 / Gestion de la reproduction}

\section{1 / L'identification}

L'identification des animaux dans le cadre de la gestion de la reproduction trouve ses applications directes dans la connaissance de l'ascendance des individus, afin d'éviter les accouplements consanguins, et dans le suivi de leurs performances zootechniques. Plusieurs systèmes peuvent être proposés mais seuls les systèmes individuels permettent un suivi personnalisé des performances (tableau 1).

Des boucles auriculaires pré-numérotées sont facilement posées à l'oreille des animaux à l'aide d'une pince. II en existe plusieurs modèles (photo 2). Compte tenu de la

Tableau 1. Systèmes d'identification en aulacodiculture.

\begin{tabular}{|c|c|c|l|l|} 
& Suivi du lignage & Suivi des performances & Avantages & Inconvénients \\
\hline $\begin{array}{c}\text { Boucles } \\
\text { auriculaires }\end{array}$ & ++ & oui & $\begin{array}{l}\text { pose } \\
\text { lisibilité }\end{array}$ & $\begin{array}{l}\text { perte possible } \\
\text { coût }\end{array}$ \\
\hline $\begin{array}{c}\text { Encoches } \\
\text { auriculaires }\end{array}$ & + & oui ou non & $\begin{array}{l}\text { réalisation } \\
\text { lisibilité }\end{array}$ & $\begin{array}{l}\text { application délicate voire impos- } \\
\text { sible pour des cheptels importants }\end{array}$ \\
\hline $\begin{array}{c}\text { Gestion } \\
\text { spatiale }\end{array}$ & + non & gratuité & $\begin{array}{l}\text { difficulté de créer de nouveaux } \\
\text { groupes de reproduction }\end{array}$ \\
\hline $\begin{array}{c}\text { Tatouage } \\
\text { Puces } \\
\text { électroniques }\end{array}$ & ++ & oui & indélébile & $\begin{array}{l}\text { réalisation } \\
\text { lisibilité }\end{array}$ \\
\hline \multirow{2}{*}{++} & oui & $\begin{array}{l}\text { indélébile } \\
\text { inviolable } \\
\text { lecture } \\
\text { immédiate }\end{array}$ & coût \\
\hline
\end{tabular}


finesse de la peau des oreilles des aulacodes, les modèles légers en plastique sont plus adaptés pour les jeunes individus. Toutefois, leur lecture est rendue malaisée chez les adultes par les poils qui se développent dans les oreilles, et les modèles métalliques, plus lourds, sont alors plus pratiques. C'est un système individuel, facile à poser et à lire, et qui convient parfaitement pour des effectifs importants.

Des encoches de forme géométrique peuvent être effectuées aux oreilles des animaux. Elles reprendront les caractéristiques de celles des parents, et plusieurs systèmes de codification peuvent être conçus, compte tenu de la possibilité de combiner plusieurs formes, plusieurs marques sur une même oreille, et de marquer une ou les deux oreilles (figure 2). Ce système s'applique au suivi du lignage des individus, mais devient vite difficile à maîtriser lorsque le cheptel est important.

Les encoches peuvent aussi être utilisées afin de traduire une identité chiffrée (figure 3). Mais ce système ne convient qu'à des petits effectifs compte tenu de la petite taille des oreilles et du nombre limité de combinaisons.

La gestion spatiale du lignage consiste à attribuer un secteur déterminé de l'élevage à tous les animaux issus d'un même groupe de reproduction. Dans le moyen terme, ce système trouve ses limites dans la constitution de nouveaux groupes de reproduction issus de lignages différents.

Bien que délicat à appliquer à l'aulacode dont la peau est extrêmement fragile, le tatouage peut se réaliser sur la face interne de la cuisse avec du bon matériel et en appliquant préalablement de la vaseline. Toutefois, le pelage de l'aulacode complique sa lecture et sa réalisation nécessite une anesthésie.

Les puces électroniques placées sous la peau constituent un système individuel idéal compte tenu de sa durabilité. Toutefois son coût prohibitif en comparaison de la valeur des animaux reste un frein à son utilisation.

\section{2 / Les critères de constitution d'un groupe de reproduction}

Les débuts de l'aulacodiculture ont été particulièrement difficiles, notamment du fait d'une mauvaise maîtrise de la reproduction en captivité de cette espèce. Si aujourd'hui, sa domestication en cours permet une meilleure expression des aptitudes reproductrices en captivité, un certain nombre de règles restent néanmoins incontournables afin d'optimiser la reproduction des aulacodes en élevage.

\section{Age et puberté}

Le choix des reproducteurs tient compte tout d'abord de la maturité sexuelle des animaux. Si les femelles sont nubiles dès 5 mois, voire dès 4 mois pour certaines, nous recommandons vivement d'attendre l'âge de 6 mois avant de les mettre à la reproduction, et de s'assurer que leur poids est de $1800 \mathrm{~g}$ au mini-
Photo 2. Pinces pour la pose des boucles auriculaires.

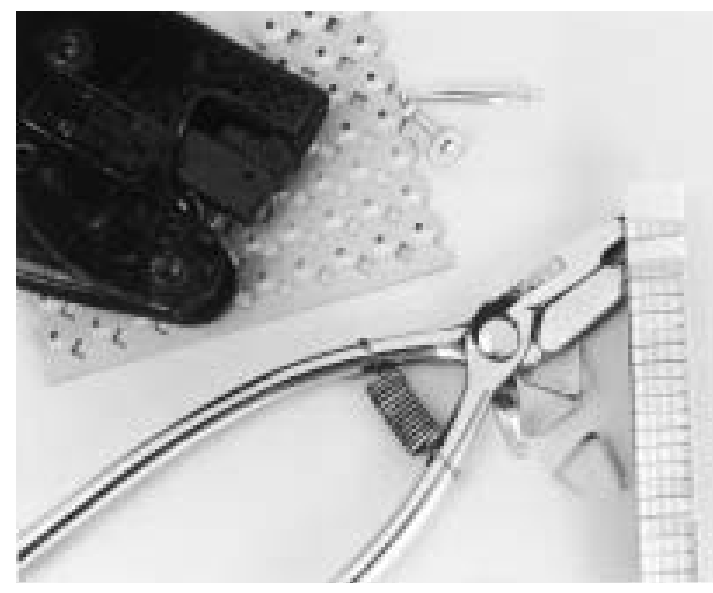

Figure 2. Encoches auriculaires géométriques.

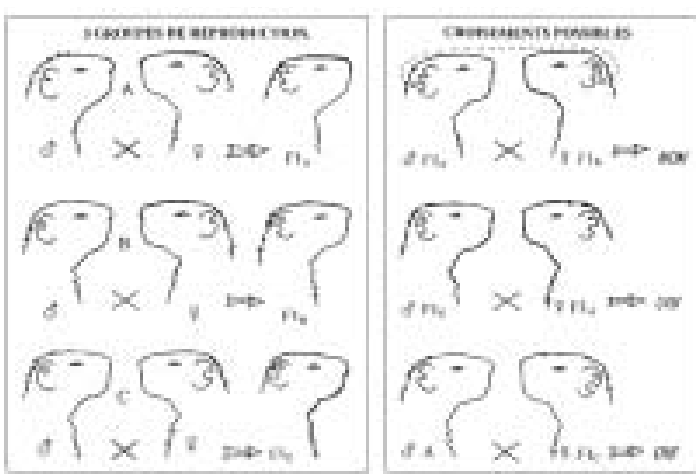

Figure 3. Encoches auriculaires codées.
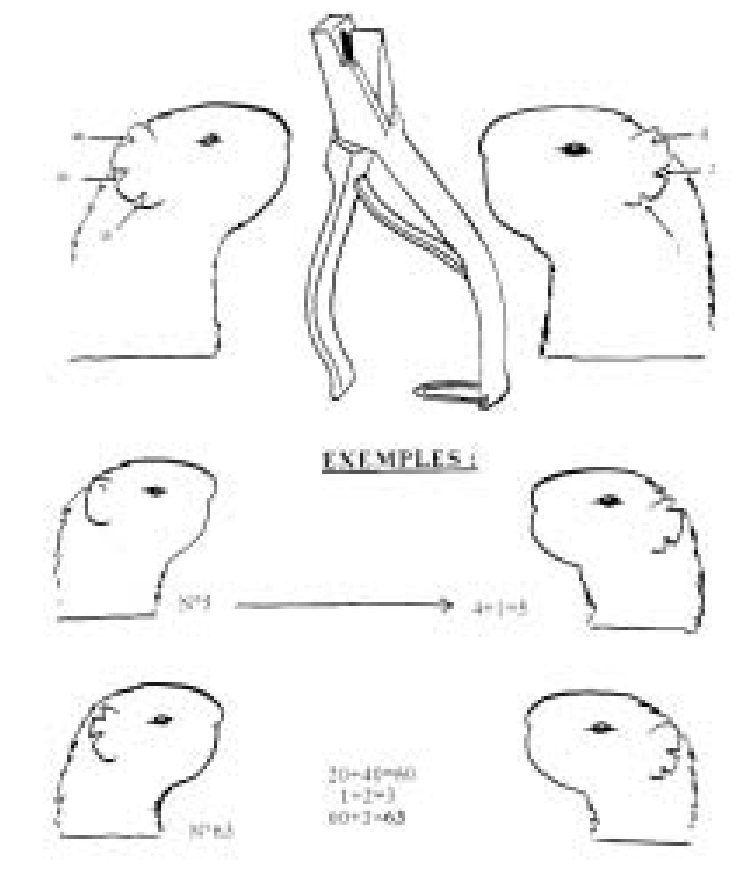

Maîtriser la reproduction nécessite d'abord d'identifier les animaux. Les boucles auriculaires semblent le moyen le plus adapté a ux aulacodes. 
mum, soit $72 \%$ du poids de la femelle adulte. En effet, la prolificité de la première portée est diminuée si la mise à la reproduction est trop précoce, avec moins de 4 petits par portée en moyenne ( $D$. Edderai, résultats non publiés), et la mise en présence de femelles de trop petit gabarit avec un mâle les expose à des blessures qui peuvent être mortelles (écart de poids important entre le mâle et les femelles, énervement du mâle face à des femelles peu réceptives, etc.). Chez les mâles, la puberté, qui se traduit par une coloration brunâtre de la zone génitale, est observée dès l'âge de 4 à 6 mois. Toutefois, nous recommandons de ne pas mettre les mâles en accouplement avant l'âge de 8 mois car, plus tôt, l'écart de poids qu'ils ont avec les femelles reste encore faible et le taux de réussite des accouplements peut être diminué.

\section{Poids}

Même s'il est possible d'effectuer des accouplements en couples monogames, il est bien plus intéressant économiquement de pratiquer les accouplements en groupes polygames, puisque cela permet de diminuer la proportion de mâles géniteurs dans le cheptel. Des groupes comportant jusqu'à 6 femelles pour un mâle donnent de très bons résultats avec un taux de gestation de $95 \%$ (DGE 1999 et 2000).

Au sein du groupe, nous recommandons que le poids du mâle dépasse celui des femelles de 1000 à $2000 \mathrm{~g}$. Si l'écart de poids est trop faible, les femelles se dérobent à la monte, ce qui conduit à des infertilités apparentes. Si le mâle est trop lourd, la saillie risque d'être violente. Le groupe de femelles doit être le plus homogène possible afin d'éviter les bagarres entre femelles et les phénomènes de compétition dans l'accès à la nourriture. Nous recommandons un écart de poids maximum de $500 \mathrm{~g}$ entre les femelles.

\section{Conditions de réalisation de l'accouplement}

L'accouplement est précédé d'une parade amoureuse qui déclenche l'œstrus chez les femelles (Adjanohoun 1992). Cette cour assidue que le mâle doit faire aux femelles nécessite de l'espace. C'est pourquoi la reproduction des aulacodes se fait en enclos ou dans des grandes cages aménagées à cet effet : $2 \mathrm{~m}^{2}$ pour un groupe de 6 femelles et un mâle, et une surface minimum de $0,5 \mathrm{~m}^{2}$ pour un couple.

Le transfert du mâle au sein d'un groupe de femelles le rend dangereusement agressif et il est alors nécessaire de le placer au sein de l'enclos dans une cage de contention d'où on ne le libérera qu'après 72 heures (Mensah et Baptist 1986). Il est nettement préférable de transférer toutes les femelles ensemble dans l'enclos du mâle. Le transfert groupé des femelles évite les bagarres, et le mâle qui se sent dans son territoire fait une cour assidue aux femelles ce qui favorise une entrée en chaleur rapide et synchronisée des aulacodines.

\section{3 / Modes de mise à la reproduction}

\section{Mise en accouplement temporaire}

La mise en accouplement temporaire consiste à isoler progressivement dans un groupe de reproduction toutes les femelles gestantes. Elle implique donc d'effectuer un test de gestation et de reformer les groupes à chaque nouvelle mise à la reproduction , qui peut s'opérer immédiatement après le sevrage des jeunes, ou après une période de repos post-sevrage de 15 jours.

Cela permet :

- l'entrée en chaleur rapide des femelles lors de la mise en présence du mâle et l'obtention de saillies précoces et synchronisées qui conduisent a des mise bas sur une courte période. A la station de multiplication du DGEG, où n'est pratiqué que l'accouplement temporaire, $92 \%$ des femelles gestantes $(n=219)$ sont saillies dans les deux premières semaines d'accouplement. II en resulte une commodité dans la constitution, après le sevrage, de groupes d'engraissement homogènes :

- le dépistage précoce des individus inféconds grâce à la pratique systématique du diagnostic de gestation

- l'utilisation intensive des mâles, un même mâle pouvant servir au sein de plusieurs groupes ;

- la diminution des risques d'infanticide ;

- la connaissance stricte de la prolificité des reproductrices et de l'ascendance maternelle des nouveau-nés, impérative en station de multiplication :

- un choix compatible des géniteurs du fait de la reconstitution systématique des groupes de reproduction.

Toutefois, la mise à la reproduction temporaire a des inconvénients :

- l'intervalle mise bas - saillie fécondante est augmenté compte tenu de l'isolement des femelles pendant I'allaitement. Ainsi, en pratiquant le sevrage à 30 jours, le nombre théorique maximum de mise bas par femelle et par an est de 2 ;

- l'isolement des femelles nécessite plus d'infrastructures, ce qui a un coût :

- la programmation du diagnostic de gestation et les manipulations qu'implique sa pratique peuvent être une contrainte pour l'éleveur.

\section{La mise en accouplement permanent}

Pour ce mode de mise à la reproduction, le groupe est formé une fois pour toutes, les femelles mettent bas dans l'enclos en présence du mâle et y élèvent leurs petits. La seule intervention de l'éleveur se limite au retrait des jeunes au sevrage. Dans la pratique, en général, l'éleveur n'effectue pas le test de gestation.

L'accouplement permanent présente plusieurs avantages:

- simplicité dans la gestion du cheptel, l'éleveur n'étant plus contraint d'isoler les femelles :

- réduction du coût des infrastructures et un 
gain d'espace

- diminution de l'intervalle mise bas - saillie fécondante du fait de l'existence d'un œstrus post-partum, avec possibilité d'obtenir en théorie au maximum 2,4 mise bas par femelle et par an. En pratique, les femelles sont fréquemment saillies dans les 15 jours suivant la mise bas, et le test de gestation effectué 10 jours après le sevrage est généralement positif ;

- possibilité d'adoption des jeunes par d'autres femelles du groupe si leur mère meurt.

Les inconvénients sont les suivants :

- la détermination de l'ascendance maternelle et de la prolificité de chaque femelle peuvent être rendues impossibles si plusieurs mise bas ont lieu en même temps ;

- on risque au fil du temps un étalement des mise bas au sein d'un même groupe, qui compliquera la constitution de lots d'engraissement homogènes. En effet, en accouplement permanent, on perd l'effet de synchronisation des accouplements induit par l'apport groupé des femelles au mâle :

- le séjour permanent du mâle dans l'enclos de reproduction n'intensifie pas son utilisation ;

- les risques d'infanticide existent, même s'ils sont rares. Dans les essais menés au DGEG, nous n'avons jamais constaté d'infanticide exercé par le mâle, mais toujours par une femelle du groupe $(4,1 \%$ des femelles ; $n=48$ ) qui peut tuer et/ou dévorer les nouveau-nés d'autres femelles, tout comme les siens. Ces femelles sont à réformer du cheptel reproducteur. Afin de limiter les risques d'infanticide, il est indispensable que les animaux soient suffisamment nourris, et que le nombre de mangeoires dans l'enclos soit suffisant pour éviter les phénomènes de compétition dans l'accès à la nourriture :

- le dépistage des individus inféconds est tardif et aléatoire.

\section{Propositions de modèles mixtes}

Si la productivité des femelles semble mathématiquement accrue dans l'accouplement permanent, il faut relativiser cette donnée. En effet, nous avons constaté que les femelles ne sont pas toujours immédiatement saillies après la mise bas, la diminution de l'intervalle mise bas - saillie fécondante associée à la mise en accouplement permanent n'est donc pas systématique. A la station du DGEG, cela a été observé pour 2 femelles sur 19 ayant mis bas en présence du mâle (soit $5,2 \%$ des cas). L'une accusait un retard de 4 semaines, et l'autre de 8 semaines. Ce décalage (retard du moment de l'accouplement) peut conduire à terme à un étalement des mise bas au sein d'un même groupe, qui va compliquer la constitution de lots d'engraissement homogènes (poids, taille). L'éleveur devra alors augmenter les infrastructures consacrées aux jeunes à l'engraissement, ce qui diminuera partiellement le gain obtenu en se dispensant de l'isolement des femelles gestantes.

Dans le cas de la mise en accouplement permanent, il peut aussi arriver que des femelles ayant pris du poids ne se laissent plus saillir (phénomène rencontré au DGEG pour une femelle sur 19). Cela conduit bien sûr à une baisse de la productivité de la femelle qui aurait pu être compensée en mise en accouplement temporaire par le réaménagement systématique des groupes.

Enfin, même si cela n'a pas encore été mesuré, un effet d'épuisement des femelles, particulièrement des plus performantes, pourrait se manifester suite à l'absence de période de repos lors de la mise en accouplement permanent (perte de poids, baisse de prolificité, mauvaise lactation). Cependant, des observations effectuées en station sur 12 femelles, et sur seulement 2 rangs de mise bas successifs, il ressort qu'aucun paramètre zootechnique ( poids des femelles, taille de la portée, poids de la portée au sevrage) n'a été affecté par l'enchaînement des gestations et la conduite simultanée de l'allaitement et de la gestation. Ceci pourrait être lié en partie à certaines caractéristiques de l'embryologie des Hystricomorphes, à savoir de longues durées de gestation et une croissance fœtale lente (Roberts et Perry 1974).

A la station de multiplication du DGEG, où nous ne pratiquons que l'accouplement temporaire, le taux de fertilité apparent, c'est-àdire le nombre de mise bas rapporté au nombre de femelles mises en accouplement est de $94 \%(n=233)$ et $92 \%$ des femelles sont saillies dans les 4 premières semaines. Ainsi, si l'application d'un schéma strict d'amélioration des performances zootechniques, tel que pourrait se l'imposer une station de multiplication, nécessite la pratique de l'accouplement temporaire pour la maîtrise parfaite des données génétiques et zootechniques (prolificité, aptitude à être saillie rapidement suite à la mise en présence du mâle) des individus, il pourrait être proposé dans le cadre de ce mode de mise à la reproduction d'intensifier I'utilisation des mâles en ne les laissant que 4 semaines avec les femelles.

L'efficacité de la mise à la reproduction permanente pourrait être améliorée par :

- une meilleure gestion de l'infécondité par la pratique systématique du test de gestation dès la 6ème semaine après la mise bas :

- le retrait du mâle 4 semaines après la précédente mise bas et sa réintroduction dans le groupe deux semaines avant les prochaines mise bas pour intensifier l'utilisation des mâles géniteurs tout en bénéficiant de la saillie post-partum.

Enfin, les risques soulevés quant à la constitution de lots d'engraissements homogènes n'ont pas lieu d'être dans des cheptels fonctionnant avec plus de 10 groupes de reproduction. En effet, dans ce cas, il sera toujours possible d'obtenir, au sein des différents groupes, suffisamment de naissances sur un même laps de temps pour constituer des lots homogènes.

\section{4 / Le diagnostic de gestation}

Le diagnostic de gestation est une pratique indispensable pour une bonne gestion de la

\section{L 'éleva ge en grou- pe où I'accouple- ment est perma- nent simplifie la gestion du cheptel, mais ne permet pas de bien maîtriser la reproduction individuelle.}


reproduction. II permet notamment de détecter rapidement les femelles gravides afin de leur appliquer les mesures propres à leur état, programmer les mise bas d'avance et tenir un état prévisionnel de son cheptel, déceler précocement les individus stériles et inféconds, prévoir la gestion de l'occupation des locaux.

Chez l'aulacode, trois techniques sont actuellement utilisables en pratique pour apprécier la gestation.

\section{L'appréciation du volume abdominal}

Le diagnostic de la gestation peut se faire à

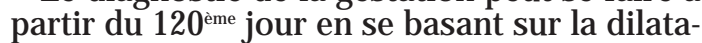
tion de l'abdomen (Adjanohoun 1988). C'est une méthode tardive et peu fiable. D'une part, l'état d'embonpoint peut se traduire chez certaines femelles par une distension de l'abdomen et, d'autre part, en cas de gestation avec seulement un ou deux foetus, la distension reste insignifiante jusqu'à la mise bas.

\section{La palpation abdominale}

Le diagnostic est fiable à partir de 45 jours de gestation (Adjanohoun 1988), mais son application nécessite l'usage d'une anesthésie afin de pouvoir manipuler correctement I'animal. Cette technique ne peut donc être vulgarisée dans les conditions d'élevage.

\section{L'examen du mucus vaginal}

Cette méthode permet de faire un diagnostic avec une fiabilité très élevée dès le 30ème jour de gestation (Adjanohoun 1988, Schrage et Togbe Yewadan 1995). En effet, il se produit dès lors des saignements dans les cornes utérines qui proviennent de la résorption embryonnaire consécutive à la chute de la progestéronémie (Adjanohoun 1988). Ces saignements viennent al ors colorer la membrane et le mucus vaginal. L'observation d'un mucus de couleur rouge vif, indique un saignement récent et donc une gestation d'environ 30 jours tandis qu'une couleur brun clair traduit un saignement plus ancien et une gestation de plus 45 jours. L'inspection du mucus doit se faire impérativement entre le 30ème et le 60ème jour de gestation car au-delà, les modifications de coloration du mucus ne sont plus visibles.

En pratique, c'est cette dernière méthode qui, compte tenu de sa précocité, de sa fiabilité et de sa simplicité, doit être retenue. Ce test peut se pratiquer à partir de la 4ème semaine de mise en accouplement. Au DGE G, afin de limiter la manipulation des animaux, il est pratiqué systématiquement sur toutes les femelles mises en accouplement à partir de la 6ème semaine. Après avoir placé la femelle dans une cage de contention, on procède d'abord à une déchirure de la membrane vaginale en imprimant, avec les doigts, un mouvement de traction vers le bas sur la vulve. Puis un coton-tige sec est introduit dans le vagin, d'abord vers le plafond puis horizontalement jusqu'au 2/3 de sa longueur en le faisant tourner entre les doigts. E n effet, en dehors de la phase de coït, I'ouverture de la vulve se situe en dessous du détroit postérieur du bassin (Adjanohoun 1992). Le cotontige est enfin ressorti et l'on observe la colo- ration du mucus.

S'il est rouge, rose ou brun, le test est positif et la femelle est gestante. Si le mucus est blanchâtre ou jaunâtre, le test est négatif. Cela ne signifie pas obligatoirement que la femelle est non gestante, mais en tout état de cause, que l'on se situe avant le 30ème jour de gestation (ou après le 60ème si l'on a oublié de programmer le test dans les 8 premières semaines après la mise en accouplement).

Si le test est négatif, la femelle reste avec le mâle et le test est refait toutes les deux semaines jusqu'à ce qu'il soit positif. Si au bout de la 12ème semaine d'accouplement, c'est-à-dire au 4ème test, celui-ci est toujours négatif, le couple est séparé ; I'accouplement a échoué. Cela peut traduire une stérilité de la femelle, mais la plupart du temps, il s'agit plutôt d'une incompatibilité entre le mâle et la femelle, et cette femelle remise en accouplement avec un autre mâle a toutes les chances de devenir gestante. Si aucune des femelles d'un groupe de reproduction n'est gestante au second test (8ème semaine d'accouplement), le mâle est changé.

Cette pratique de diagnostic de gestation qui est invasive et septique est parfaitement tolérée par les femelles. Aucun cas d'infection utérine ou vaginale n'a jamais été engendré par cette opération.

Enfin, les résultats du DGEG (2000) donnent un sensibilité du test négatif égale à $100 \%(n=14)$ car nous n'avons jamais été confrontés à une femelle qui a mis bas après avoir eu plusieurs tests de gestation négatifs. De même, la sensibilité du test positif est de $100 \%(n=219)$ et toutes les femelles ayant eu un test positif ont mis bas. Toutefois, des cas de femelles ayant eu un test positif et n'ayant jamais mis bas ont été rencontrés chez des éleveurs. Ces cas s'expliquent probablement par des avortements.

\section{Conclusion}

L'adoption de certaines pratiques s'avère indispensable à une bonne gestion de la reproduction et/ou à une optimisation de la productivité des élevages. Les points qui nous semblent les plus importants sont les suivants:

- l'identification : elle est indispensable pour une bonne connaissance du lignage des individus et ainsi éviter les phénomènes de consanguinité. Parmi tous les systèmes précités, nous recommandons l'usage des boucles prénumérotées pour tous les individus d'une station de multiplication ou de sélection. Dans le cadre d'un élevage productif, l'identification des seuls géniteurs et futurs géniteurs s'avère utile ;

- la pratique du test de gestation par examen du mucus vaginal : cette technique est d'une parfaite fiabilité, facile à réaliser et précoce. $\mathrm{Par}$ conséquent, nous recommandons vivement la généralisation de sa pratique quel que soit le mode d'accouplement pratiqué, afin notamment de permettre une bonne gestion de l'infécondité et des pertes économiques 
qu'elle engendre ;

- enfin, pour une bonne gestion de la reproduction, une station de multiplication et encore plus une station de sélection doit pratiquer
I'accouplement temporaire, I'accouplement permanent étant lui adapté aux éleveurs qui pourront au fur et à mesure de leur spécialisation adopter le test de gestation.

\section{Références}

Adjanohoun E., 1988. Contribution au développement de l'élevage de l'aulacode (Thryonomys swi nderianus Temminck, 1827) et à l'étude de sa reproduction. Thèse doc. vét., Créteil, 199 p.

Adjanohoun E., 1992. Le cycle sexuel et la reproduction de l'aulacode (Thryonomys swinderianus Temminck, 1827). Mammalia, 56, 109-119

DGEG, 1999. Rapport d'activité annuel, 56 p. Vétérinaires sans frontières, Lyon.

DGEG, 2000. Rapport d'activité annuel, 48 p. Vétérinaires sans frontières, Lyon.

Heymans J.C., 1996. L'élevage de l'aulacode. Cahier Conservation. $n^{\circ} 31$. FAO, Rome, 98 p.

J ori F., Mensah G.A., Adjanohoun E., 1995. Grasscutter farming: an example of rational utilization of wildlife. Biodiversity and Conservation, 4, 257-265.

Jori F., Noel J.M., 1996. Guide pratique d'élevage d'aulacodes au Gabon. Vétérinaires sans frontières / Coopération Française, $55 \mathrm{p}$.
Kingdon J., 1997. The Kingdon fiels guide to african mammals. Academic Press Natural World, San Diego, USA, $188 \mathrm{p}$.

Mensah G.A., Baptist R., 1986. Aspects pratiques en élevage d'aulacodes (Thryonomys swinderianus) I. Modes d'accouplement et durée de la gestation. Rev. Elev. Méd. vét. Pays trop., 39, 239-242.

Productions animales, 1992. L'élevage de l'aulacode. MCD / IE MVT-CIRAD, fiches techniques d'élevage tropical, fiche $n^{\circ} 2$.

Roberts C.M., Perry J.S., 1974. Hystricomorph embryology. Symp. zool. Soc. Lond., 34, 333-360.

Schrage R., Togbe Yewadan L., 1995. Abrégé d'aulacodiculture. GTZ, 103 p.

Van de Velde M., 1991. L'élevage d'aulacodes au Zaïre. Publication du service agricole n²7, Bruxelles, Belgique. $90 \mathrm{p}$

Weir B.J., 1974. Reproductive characteristics of Hystricomorph rodents. Symp. zool. Soc. Lond., 34, 265-301.

\section{Abstract}

Reproduction management in cane-rats breeding. Synthesis of the existing tools and methods.

Cane rats breeding is now technically well known and reproduction in captivity of this African rodent works well. However, in an economical logic, a good management of the reproduction is essential to enable an optimal productivity of the females. So, sires have to be identified to avoid inbreeding and select the most performant ones. Mating is made in polygamous group. Being lighter than the male, the femalesare brought to the male's pen. An easy and early gestation test by examination of the vaginal mucus color exists and should be made as routine practice. When pregnant, females can be isolated until weaning (what is called temporary mating), or left permanently with the male in the pen (permanent mating) for an early covering after birth.

EDDERAI D., NTSAME M., HOUBEN P., 2001. Gestion de la reproduction en aulacodiculture. Synthèse des outils et méthodes existants. INRA Prod. Anim., 14, 97-103. 
